\title{
EFEITO DO ETHEPHON NA QUALIDADE DA UVA 'NIAGARA ROSADA' (Vitis labrusca L.), PRODUZIDA NA ENTRESSAFRA, NA REGIÃO DE JALES-SP ${ }^{1}$
}

\author{
FERNANDO MENDES PEREIRA² \& ANTONIO AUGUSTO FRACARO ${ }^{3}$
}

\begin{abstract}
RESUMO- Na região de Jales (SP), a produção de uva 'Niagara Rosada' obtida de videiras podadas em períodos de ocorrência de baixas temperaturas é, em geral, insatisfatória, apresentando pequena produtividade e cachos com qualidade inadequada à comercialização. Essa qualidade da produção está intimamente relacionada à efetiva brotação. Assim, este trabalho teve o objetivo de avaliar a aplicação do ethephon em diferentes doses antes da poda, visando a melhorar a brotação e a qualidade dos cachos, em seis experimentos, durante os anos de 2001 e 2002. Foram testadas as doses de 0; 3; 6 e 9 L.ha $^{-1}$ de ethephon aplicado via foliar. Concluiu-se que o uso de ethephon, na dose de 9 L.ha $^{-1}$, proporcionou cachos e bagas maiores e com maiores pesos, comprimento e largura, melhorando o aspecto dos mesmos. A aplicação de ethephon não afetou os teores de sólidos solúveis totais e a acidez total titulável, não alterando o sabor da uva 'Niagara Rosada'.
\end{abstract}

Termos de indexação: Vitis sp, fitorreguladores, qualidade dos cachos, ethephon.

\section{THE EFFECT OF ETHEPHON IN THE QUALITY OF GRAPES 'NIAGARA ROSADA’ (Vitis labrusca L.) PRODUCED IN THE REGION OF JALES-SP}

\begin{abstract}
In the region of Jales (SP) the 'Niagara Rosada' grape yield obtained of grapevines pruned in periods of occurrence of low temperatures is in general unsatisfactory, presenting small yield and clusters with inadequate quality to commercialization. This quality of the yield is related to effective sprouting. Thus, this work rad the objective to evaluate the application of ethephon in different levels before the pruning, aiming the improvement of sprouting and clusters quality, in six experiments during the years of 2001 and 2002. Levels of 0, 3, 6 and 9 L.ha ${ }^{-1}$ of ethephon were applied by leaf. It was concluded that the use of ethephon, in level of $9 \mathrm{~L}^{-h^{-1}}$, provided bigger clusters and berries with greater weight, length and width, improving their aspect. The application of ethephon did not affect the tenor of total soluble solid and the titulabre total acidity, not modifying the flavor of grape 'Niagara Rosada'.
\end{abstract}

Index Terms- Vitis sp, growth regulator, cluster quality, ethephon.

\section{INTRODUÇÃo}

A ‘Niagara Rosada’ produzida na região de Jales-SP, no período de entressafra, durante os meses de setembro-outubro, alcança preços acima de $\mathrm{R} \$ 1,80 \mathrm{~kg}^{-1}$, porém essa produção encontra problemas, principalmente relacionados à dificuldade de emissão e desenvolvimento das brotações após as podas de produção realizadas quando da ocorrência de temperaturas mais baixas, normalmente entre maio e julho. Tal fato reduz a produtividade em decorrência da formação de um menor número de cachos e de cachos fora do padrão comercial, sendo os mesmos pequenos, não compactos e com poucas bagas, conseqüentemente, desestimulando os viticultores a produzir nesta época do ano.

Na tentativa de solucionar esses problemas, é comum na região a utilização de cianamida hidrogenada aplicada após a poda, em doses de até $15 \%$, e com resultados nem sempre satisfatórios.

A utilização de ethephon em videira 'Rubi', na região Noroeste do Estado de São Paulo, resultou em cachos uniformes com padrão comercial e com acidez total titulável e sólidos solúveis totais dentro da faixa exigida para comercialização (Fracaro, 2000). Além disso, a aplicação de ethephon não afetou comercialmente as características dos cachos e bagas, estando dentro dos padrões determinados por Boliani (1994), que estudou a fenologia das uvas 'Rubi e 'Itália' para a região Noroeste do Estado de São Paulo.

A aplicação de ethephon em variedades européias resultou na melhoria dos teores de sólidos solúveis totais, em trabalhos realizados por Hardie et al. (1981), Manini et al. (1981) e Larios et al. (1987). Relativamente à acidez total titulável, somente Manini et al. (1981) não observaram respostas positivas.

O objetivo deste trabalho foi de verificar o efeito de diferentes concentrações de ethephon, aplicado via foliar antes da poda de produção, sobre a qualidade da uva 'Niagara Rosada', na região Noroeste do Estado de São Paulo.

\section{MATERIAL E MÉTODOS}

Foram realizados três experimentos no ano 2001 e repetidos no ano de 2002, em vinhedos comerciais da cv Niagara Rosada, espaçados de $2,5 \times 2,0 \mathrm{~m}$ e conduzidos no sistema latada, tendo como porta-enxerto o IAC 572-Jales. Aárea experimental foi implantada em cinco propriedades, na região de Jales, Noroeste do Estado de São Paulo, situada na latitude de $20^{\circ} 16^{\prime} \mathrm{S}$, longitude $50^{\circ} 33^{\prime} \mathrm{O}$ e altitude média de $483 \mathrm{~m}$,

O clima da região é classificado como CWa. A precipitação pluvial média anual é de $1.280 \mathrm{~mm}$ distribuídos principalmente durante os meses de agosto a abril. A estação seca ocorre entre os meses de maio a setembro. Apresenta evapotranspiração média anual de $2.205 \mathrm{~mm}$ e evapotranspiração de $234,1 \mathrm{~mm}$. A temperatura média anual é de $22,3^{\circ} \mathrm{C}$, com média das mínimas de $19,9^{\circ} \mathrm{C}$ e média das máximas de $29,0^{\circ} \mathrm{C}$. A umidade relativa média é de 69\%, com máxima em março (76\%) e mínima em setembro (61\%) (Boliani, 1994).

O solo predominante da região está classificado como Podzólico Vermelho-Amarelo. O relevo na região é suave-ondulado e ondulado (Terra et al., 1998).

O delineamento experimental utilizado foi em blocos ao acaso, com quatro doses de ethephon e cinco repetições, e uma planta por parcela. Os tratamentos utilizados foram: 1)Testemunha (sem aplicação de ethephon); 2) 3L.ha' ${ }^{-1}$;)6L.ha ${ }^{-1}$, e 4)9L.ha ${ }^{-1}$ do produto comercial contendo $240 \mathrm{~g} . \mathrm{L}^{-1}$ de ethephon.

Em 2001, os tratamentos foram aplicados via foliar em plantas com $30 \%$ de enfolhamento, com pulverizador costal, até o ponto de escorrimento (1.000 litros por hectare), nos dias 22-05, 30-05 e 06-06, podando-se as plantas nos dias 11-06, 18-06 e 25-06, respectivamente, para os experimentos $1 ; 2$ e 3 . No ano de 2002, os tratamentos foram aplicados com pulverizador até o ponto de escorrimento nas plantas com $80 \%$ de enfolhamento, nos dias 25-05, 06-06 e 17-06, podando-se as plantas nos dias 12-06, 22-06 e3-07, respectivamente, para os experimentos 1; 2 e 3.

\footnotetext{
${ }^{1}$ (Trabalho 036/2004). Recebido: 02/04/2004. Aceito para publicação: 21/07/2004. Parte da tese de doutorado do segundo autor a FCAV/UNESP. Trabalho financiado pela FAPESP.

${ }^{2}$ Dr., Prof. Titular Aposentado, Departamento de Produção Vegetal, FCAV/UNESP, Câmpus de Jaboticabal, Jaboticabal-SP.

${ }^{3}$ Doutorando em Agronomia, Departamento de Produção Vegetal, Universidade Estadual Paulista-UNESP, Faculdade de Ciências Agrárias e Veterinárias- FCAV, Câmpus de Jaboticabal, Rodovia de Acesso Prof. Paulo Donato Castellane, s/n, CEP14884-900, Jaboticabal-SP. Bolsista CNPq. Fone 17 3621 6884. E-mail: fracaro@melfinet.com.br.
} 
A medição do comprimento e da largura dos cachos foi realizada através de paquímetro digital de $0,01 \mathrm{~mm}$ de resolução. O peso dos cachos foi determinado através da relação: produção/número de cachos. Foram utilizadas 20 bagas por tratamento para a análise do teor de sólidos solúveis totais realizada com auxílio de refratômetro manual; e a acidez total titulável foi determinada conforme recomendações de Tressler \& Loslyn (1961). O peso médio das mesmas foi determinado em balança de precisão de dois dígitos, e comprimento e diâmetro das bagas foi feito com auxílio de paquímetro digital. Os dados foram analisados através de regressão polinomial, pelo programa SAS. Todos os tratos culturais utilizados foram os convencionais adotados para a cultura na região.

\section{RESULTADOS E DISCUSSÃO}

\section{Efeito sobre o comprimento e largura dos cachos}

Verifica-se, nas Figuras 1 e 2, clara tendência de aumento no comprimento e largura dos cachos, com o aumento da dose de ethephon.

Na Tabela 1, verificam-se os resultados estatísticos de todas as variáveis.

O comprimento e a largura dos cachos foram menores em 2001 que em 2002. Igual resposta foi verificada para a produtividade, o que sugere uma contribuição do aumento no tamanho dos cachos na produtividade.

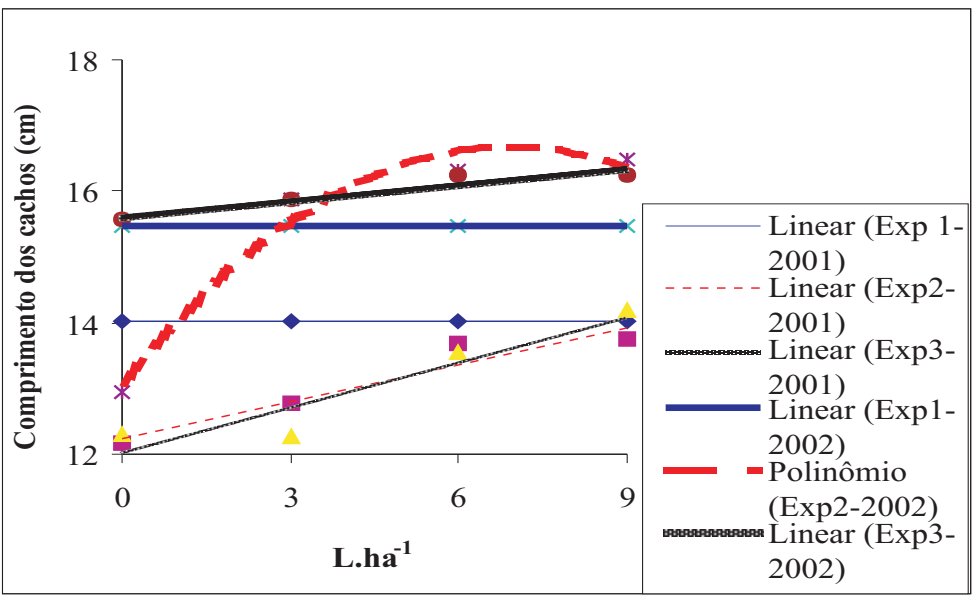

FIGURA 1 - Efeito da aplicação de ethephon antes da poda de produção sobre o Comprimento dos Cachos $(\mathrm{cm})$ da videira 'Niagara Rosada', cultivada na região Noroeste do Estado de São Paulo. Jales-SP, 2001 e 2002.

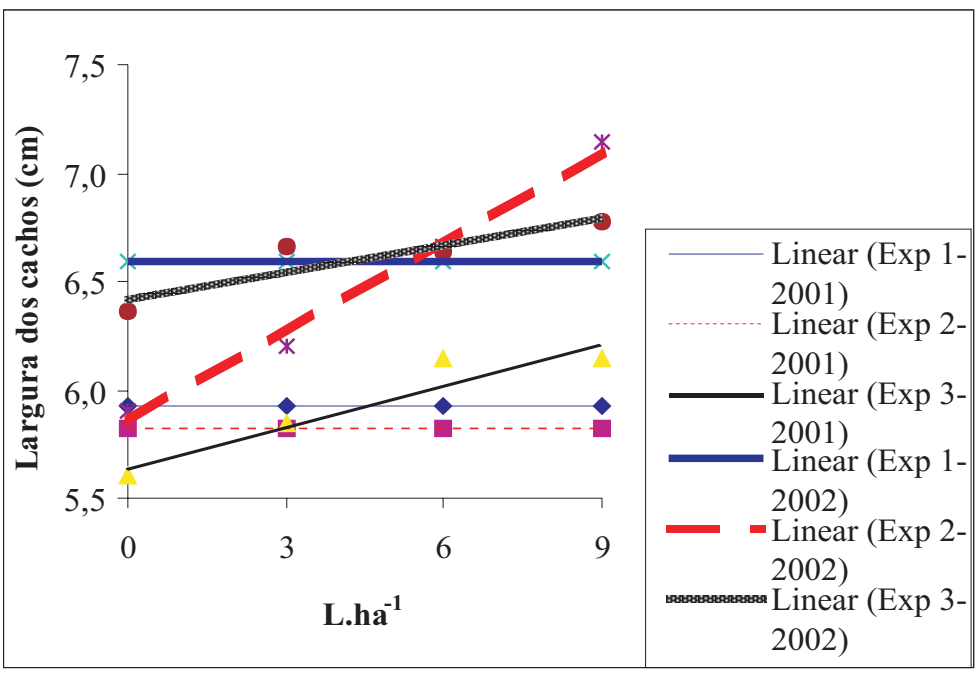

FIGURA 2 - Efeito da aplicação de ethephon antes da poda de produção sobre a Largura dos Cachos $(\mathrm{cm})$ da videira 'Niagara Rosada', cultivada na região Noroeste do Estado de São Paulo. Jales-SP, 2001 e 2002.
Portanto, fica nítida a tendência de aumento dessa variável com o aumento da dose de ethephon aplicado.

Fazendo-se uma análise conjunta dos experimentos conduzidos nos anos de 2001 e 2002, foi possível verificar que a utilização de ethephon na dose de 9L.ha- ${ }^{-1}$ é uma prática que pode auxiliar o viticultor a aumentar o comprimento e a largura dos cachos e, conseqüentemente, a produção da 'Niagara Rosada'.

\section{Efeito sobre o Peso dos Cachos}

No que diz respeito ao peso dos cachos, observa-se, na Figura 3 , a tendência de aumento nos tratamentos com a aplicação de ethephon.

Como o número de cachos do tratamento-testemunha era muito menor, os cachos remanescentes tenderam a um maior desenvolvimento. Isto devido à metodologia utilizada, na qual se considerou como peso médio dos cachos, a relação entre produção e o número total de cachos por parcela.

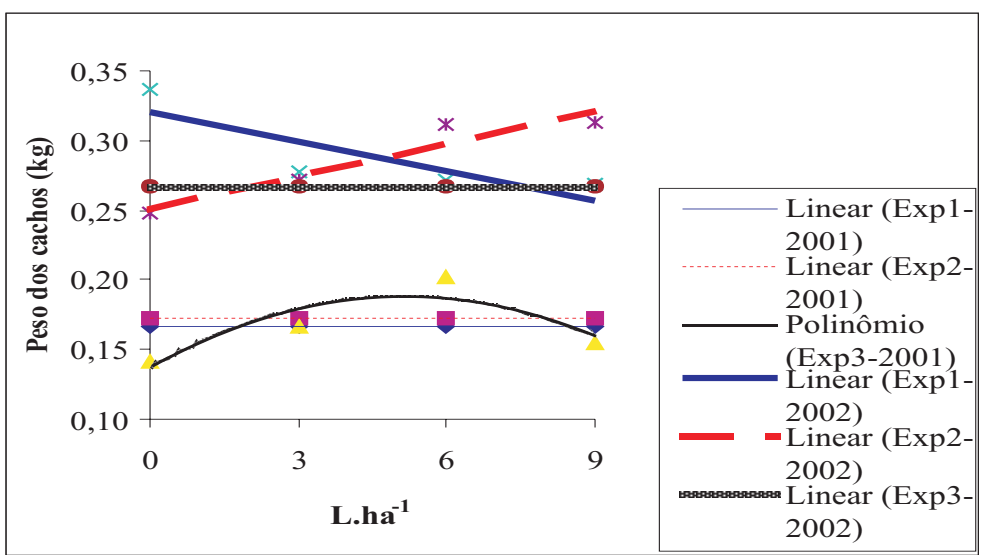

FIGURA 3 - Efeito da aplicação de ethephon antes da poda de produç̃o sobre o Peso dos Cachos (kg) da videira 'Niagara Rosada', cultivada na região Noroeste do Estado de São Paulo. Jales-SP, 2001 e 2002.

\section{Efeito sobre o Comprimento, Largura e Peso das Bagas}

Verifica-se, nas Figuras 4; 5 e 6, clara tendência de aumento no comprimento, largura e peso das bagas, com o aumento da dose de ethephon.

A análise das variáveis estudadas relativas às bagas evidencia que, nos experimentos realizados em 2002, os valores encontrados sempre se mostraram superiores aos de 2001. Tais resultados assemelham-se às variáveis peso, comprimento e largura dos cachos.

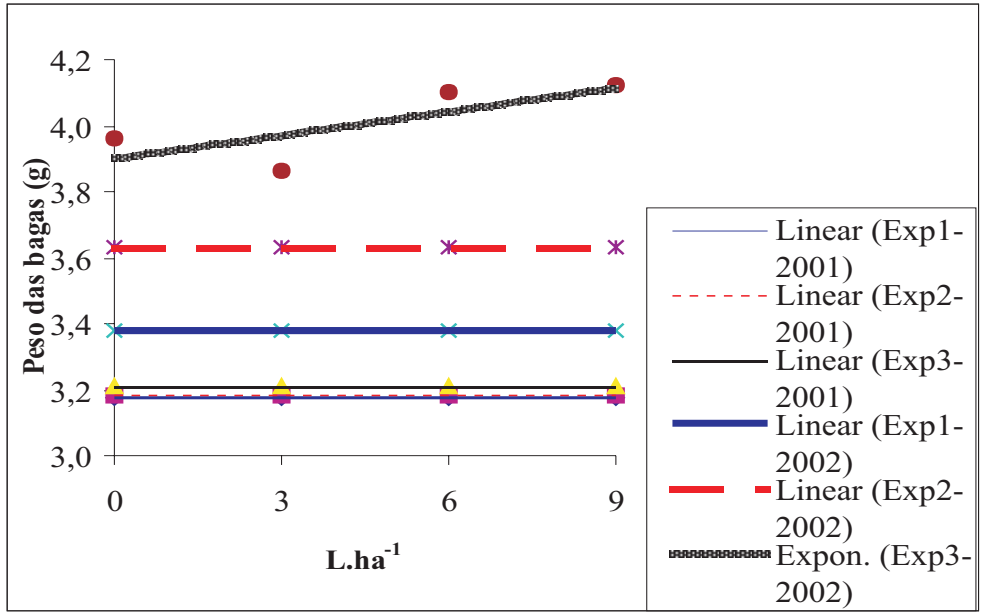

FIGURA 4 - Efeito da aplicação de ethephon antes da poda de produção sobre o Peso das Bagas (g) da videira 'Niagara Rosada', cultivada na região Noroeste do Estado de São Paulo. JalesSP, 2001 e 2002 
TABELA 1 - Resultados estatísticos da Regressão Polinomial, do efeito do ethephon na qualidade da uva 'Niagara Rosada', utilizado antes da poda de produção, na região Noroeste do Estado de São Paulo - Jales-SP, 2001 e 2002.

\begin{tabular}{|c|c|c|c|c|c|}
\hline Exp - Ano & Variável & & & $\mathbf{R}^{2}$ & Equação \\
\hline & & R.L. & R. Q. & & \\
\hline $1-2001$ & Comp. cacho & $2,23 \mathrm{~ns}$ & & 0,3851 & $\mathrm{Y}=14,03$ \\
\hline $2-2001$ & Comp. cacho & $33,7801 * *$ & & 0,9121 & $Y=0,173 x+12,252$ \\
\hline $3-2001$ & Comp. cacho & $24,5742 * *$ & & 0,8808 & $Y=0,2287 x+12,046$ \\
\hline $1-2002$ & Comp. cacho & $0,7673 \mathrm{~ns}$ & & 0,2069 & $Y=15,47$ \\
\hline $2-2002$ & Comp. cacho & & $7,9770 *$ & 0,9703 & $Y=-0,0756 x^{2}+1,0467+13,07$ \\
\hline $3-2002$ & Comp. cacho & $6,4991 *$ & & 0,8993 & $Y=0,0833 x+15,61$ \\
\hline $1-2001$ & Larg. cacho & $2,9922 \mathrm{~ns}$ & & 0,5408 & $Y=5,93$ \\
\hline $2-2001$ & Larg. cacho & $2,9922 \mathrm{~ns}$ & & 0,5408 & $Y=5,82$ \\
\hline $3-2001$ & Larg. cacho & $10,800 * *$ & & 0,8982 & $Y=0,064+5,642$ \\
\hline $1-2002$ & Larg. cacho & $0,5982 \mathrm{~ns}$ & & 0,5182 & $Y=6,59$ \\
\hline $2-2002$ & Larg. cacho & $25,2127 * *$ & & 0,9897 & $Y=0,1393 x+5,848$ \\
\hline $3-2002$ & Larg. cacho & $7,1963 *$ & & 0,8110 & $Y=0,0413 x+6,424$ \\
\hline $1-2001$ & Peso cacho & $0,008 \mathrm{~ns}$ & & 0,0034 & $\mathrm{Y}=0,16695$ \\
\hline $2-2001$ & Peso cacho & $0,1381 \mathrm{~ns}$ & & 0,0257 & $\mathrm{Y}=0,17245$ \\
\hline $3-2001$ & Peso cacho & & $7,6161^{*}$ & 0,7736 & $Y=-0,002 x^{2}+0,02053 x+0,1366$ \\
\hline $1-2002$ & Peso cacho & $6,3237^{*}$ & & 0,7836 & $Y=-0,025 x+0,3037$ \\
\hline $2-2002$ & Peso cacho & $9,9884 * *$ & & 0,9116 & $Y=0,007646667 x+0,2532$ \\
\hline $3-2002$ & Peso cacho & $2,5429 \mathrm{~ns}$ & & 0,6280 & $Y=0,26665$ \\
\hline $1-2001$ & Comp. baga & $35,2914 * *$ & & 0,9449 & $\mathrm{Y}=0,114 \mathrm{x}+16,399$ \\
\hline $1-2002$ & Comp. baga & & $9,60 * *$ & 0,9240 & $Y=-0,01111 x^{2}+0,26 x+18,63$ \\
\hline $2-2002$ & Comp. baga & $4,8461 *$ & & 0,8040 & $Y=0,0686667 x+19,996$ \\
\hline $3-2002$ & Comp. baga & $1,7655 \mathrm{~ns}$ & & 0,1994 & $Y=19,4955$ \\
\hline $3-2001$ & Larg. baga & $7,8852 *$ & & 0,8162 & $Y=0,0953 x+16,236$ \\
\hline $1-2002$ & Larg. baga & $60,4094 * *$ & & 0,9066 & $Y=0,148 x+16,494$ \\
\hline $2-2002$ & Larg. baga & $1,3889 \mathrm{~ns}$ & & 0,8424 & $Y=17,2115$ \\
\hline $3-2002$ & Larg. baga & $39,4958 * *$ & & 0,8846 & $Y=0,0893 x+17,168$ \\
\hline $1-2001$ & Peso baga & $0,1359 \mathrm{~ns}$ & & 0,3314 & $\mathrm{Y}=3,1765$ \\
\hline $2-2001$ & Peso baga & $4,3172 \mathrm{~ns}$ & & 0,9465 & $Y=3,179$ \\
\hline $3-2001$ & Peso baga & $2,7945 \mathrm{~ns}$ & & 0,9679 & $Y=3,208$ \\
\hline $1-2002$ & Peso baga & $0,0004 \mathrm{~ns}$ & & 0,0001 & $Y=3,3785$ \\
\hline $2-2002$ & Peso baga & $3,4505 \mathrm{~ns}$ & & 0,9324 & $Y=3,635$ \\
\hline $3-2002$ & Peso baga & $7,1014 *$ & & 0,5735 & $Y=0,024 x+3,902$ \\
\hline $1-2001$ & S.S.T. & $2,5584 \mathrm{~ns}$ & & 0,6997 & $\mathrm{Y}=12,495$ \\
\hline $3-2001$ & S.S.T. & $1,4388 \mathrm{~ns}$ & & 0,3241 & $Y=16,615$ \\
\hline $1-2002$ & S.S.T. & $6,5168 *$ & & 0,8475 & $Y=-0,132 x+17,144$ \\
\hline $2-2002$ & S.S.T. & $0,0625 \mathrm{~ns}$ & & 0,018 & $Y=16,53$ \\
\hline $3-2002$ & S.S.T. & $0,2169 \mathrm{~ns}$ & & 0,054 & $Y=15,455$ \\
\hline $3-2001$ & A.T.T. & $9,9535 * *$ & & 0,9695 & $Y=-0,009793 x+0,522$ \\
\hline $1-2002$ & A.T.T. & $124,8073 * *$ & & 0,9764 & $Y=-0,008733 x+0,4298$ \\
\hline $2-2002$ & A.T.T. & $0,3282 \mathrm{~ns}$ & & 0,0635 & $Y=0,4435$ \\
\hline $3-2002$ & A.T.T. & $0,1057 \mathrm{~ns}$ & & 0,8345 & $Y=0,652$ \\
\hline
\end{tabular}

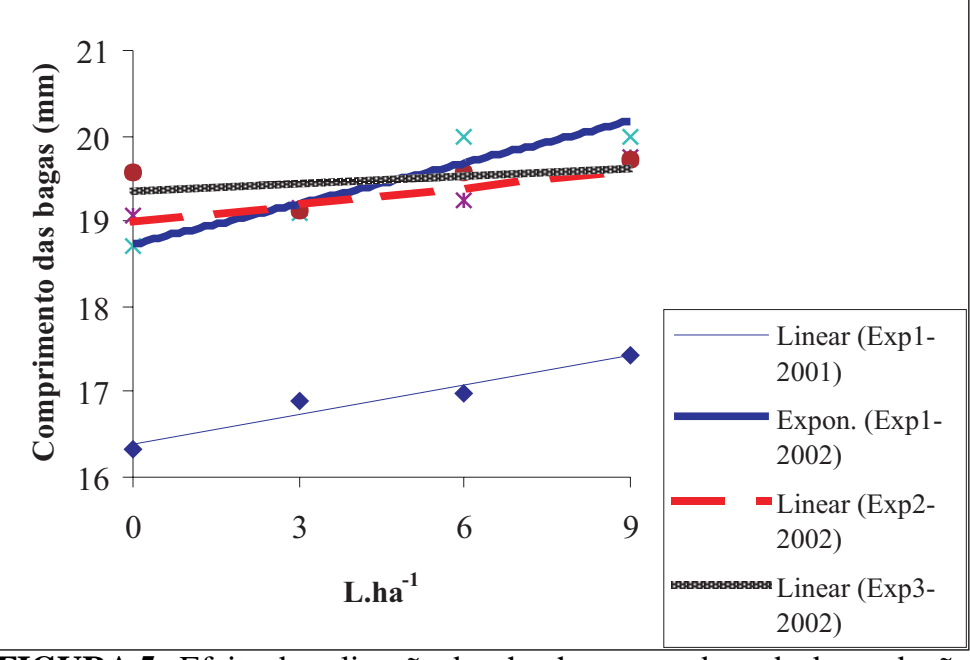

FIGURA 5 - Efeito da aplicação de ethephon antes da poda de produção sobre o Comprimento das Bagas ( $\mathrm{mm}$ ) da videira 'Niagara Rosada', cultivada na região Noroeste do Estado de São Paulo. Jales-SP, 2001 e 2002.

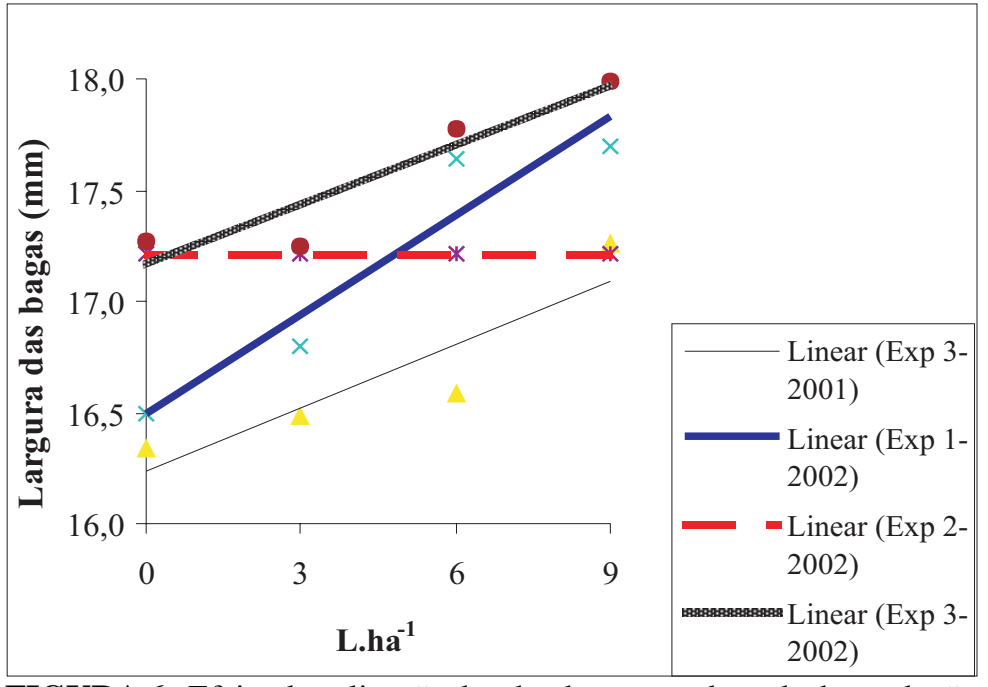

FIGURA 6 - Efeito da aplicação de ethephon antes da poda de produção sobre a Largura das Bagas (mm) da videira 'Niagara Rosada', cultivada na região Noroeste do Estado de São Paulo. Jales-SP, 2001 e 2002. 
Isto permite concluir que o aumento da produtividade foi também influenciado pelo crescimento dos cachos e das bagas.

Portanto, com a utilização de ethephon, na dose de 9 L.ha $^{-1}$, é possível obter cachos maiores com maiores bagas, propiciando a formação de cachos compactos, uniformes e mais atrativos à comercialização.

\section{Efeito sobre o teor de Sólidos Solúveis Totais}

Verifica-se, na Figura 7, a ocorrência de leve tendência à diminuição dos teores de sólidos solúveis totais com o aumento da dose de ethephon. Segundo Terra et al. (1998), as uvas apresentam características desejáveis de colheita e comercialização acima de $14^{\circ}$ Brix. Em todos os experimentos (exceção ao experimento 1 de 2001), os teores de sólidos solúveis totais apresentaram-se acima deste parâmetro, conferindo, portanto, boa qualidade à uva 'Niagara Rosada'.

Pode-se, pois, utilizar o ethephon, aplicado antes da poda de produção, sem risco de afetar significativamente os teores de sólidos solúveis totais da uva 'Niagara Rosada'.

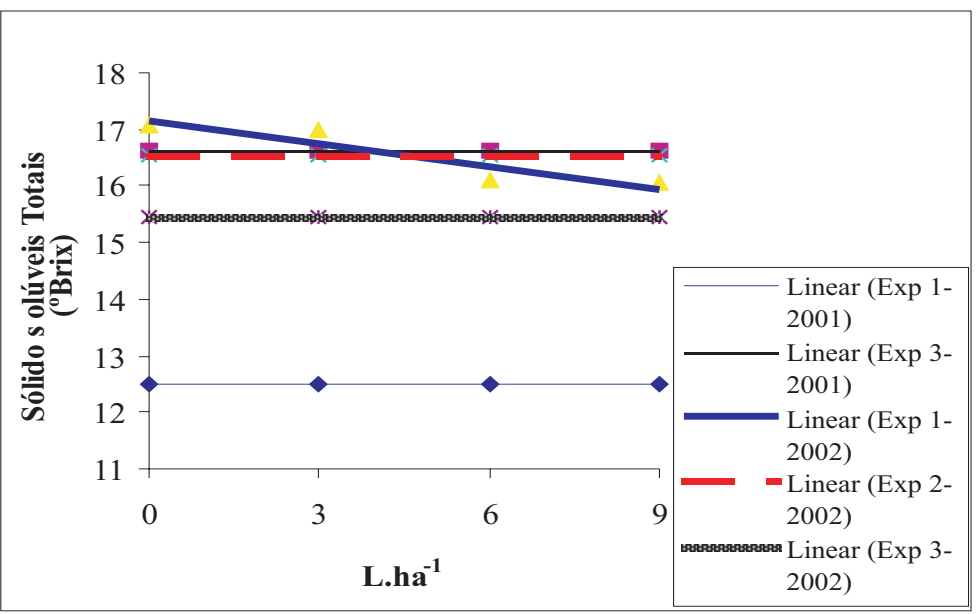

FIGURA 7 - Efeito da aplicação de ethephon antes da poda de produção sobre os teores de Sólidos Solúveis Totais ( ${ }^{\circ}$ Brix) da videira 'Niagara Rosada', cultivada na região Noroeste do Estado de São Paulo. Jales-SP, 2001 e 2002.

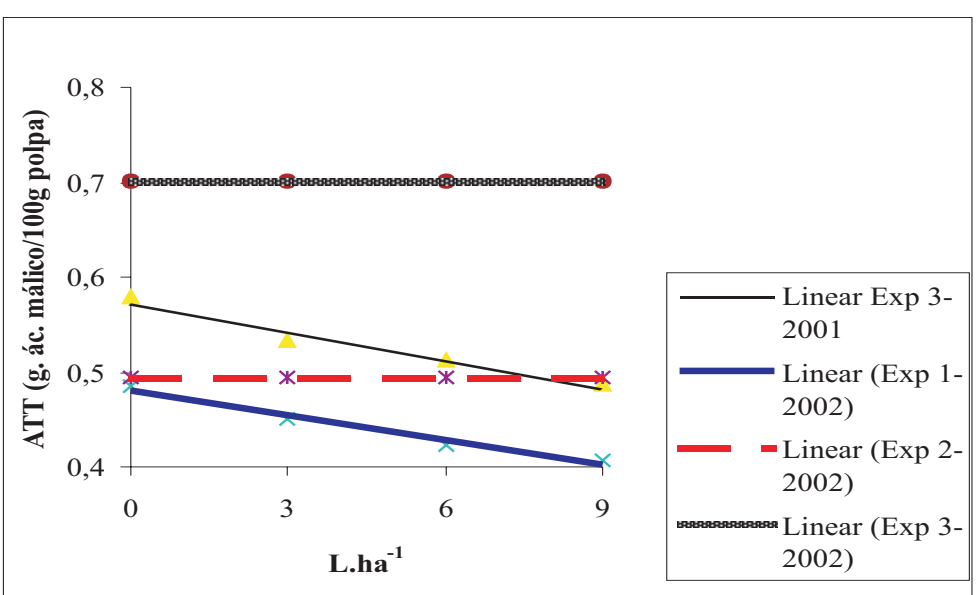

FIGURA 8 - Efeito da aplicação de ethephon antes da poda de produção sobre a Acidez Total Titulável (gramas de ácido málico por $100 \mathrm{~g}$ de polpa) da videira 'Niagara Rosada', cultivada na regiã̃o Noroeste do Estado de São Paulo. Jales-SP, 2001 e 2002

\section{Efeito sobre a Acidez Total Titulável}

Verfica-se, na Figura 8, a tendência de diminuição da acidez total titulável, com o aumento da dose de ethephon, embora os índices encontrados se apresentassem normalmente abaixo do nível máximo aceitável para comercialização. A exceção ocorreu no experimento 3 do ano de 2002, onde a colheita foi antecipada por razões comerciais. Os valores da ATT e dos SSTs obtidos apresentaram-se abaixo do desejável, indicando a colheita antes do momento adequado.

É importante ressaltar que o uso de ethephon deve estar associado ao uso adequado de outras práticas no pomar, principalmente de manejo e adubação, para otimizar a qualidade da uva 'Niagara Rosada'.

\section{CONCLUSÕES}

Nas condições em que foram desenvolvidos os experimentos, foi possível concluir que:

1. O uso de ethephon na dose de 9L.ha-1 aumentou o comprimento, a largura e o peso dos cachos e das bagas da uva 'Niagara Rosada'.

2. A aplicação de ethephon não afeta os teores de sólidos solúveis totais e a acidez total titulável.

3. O uso de ethephon, na dose de 9L.ha ${ }^{-1}$, aplicado antes da poda de produção tende a proporcionar melhor qualidade na uva 'Niagara Rosada'.

\section{REFERÊNCIAS BIBLIOGRÁFICAS}

BOLIANI, A.C. Avaliação fenológica de videiras Vitis vinifera L. cv. Itália e cv. Rubi, na região Noroeste de São Paulo. 1994. 188f. Tese (Doutorado em Produção Vegetal) - Faculdade de Ciências Agrárias e Veterinárias, Universidade Estadual Paulista, Jaboticabal, 1994.

FRACARO, A.A. Efeito de doses crescentes de ethephon em videira 'Rubi'(Vitis vinifera L.), cultivada na região Noroeste do Estado de São Paulo. 2000. 88f. Dissertação (Mestrado em Sistema de Produção) - Faculdade de Engenharia de Ilha Solteira, Universidade Estadual Paulista. Ilha Solteira, 2000.

HARDIE, W.J.; WEAVER, R.J.; JOHNSON, J.O. The influence of vine water regime on ethephon enhanced ripening of Zinfandel. American Journal of Enology and Viticulture, Davis, v.32, n.2, p. $115-21,1981$

LARIOS, G.A.; COLINAS, L.M.; SAUCEDO, V.C.; MARTINEZ, G.A. Effect of Ethrel, Alar, Cycocel and late pruning on bud break, yields and quality in grapevine (Vitis vinifera. L). Agrociência, Chapingo, v. 33, n. 68, p. 93 - 103, 1987.

MANNINI, F.; WEAVER, R.J.; JOHNSON, J.O. Effect of early bloom spray of ethephon on irrigated and non irrigated vines of Zinfadel. American Journal of Enology and Viticulture, Davis, v. 32, n.4 , p. 277-9,1981.

TERRA, M.M.; PIRES, E.J.P.; NOGUEIRA, N.A.M. Tecnologia para produção de uva 'Itália' na região Noroeste do Estado de São Paulo. Campinas:CATI, 1998. 81p. (Documento técnico, 97).

TRESSLER, D.K.; LOSLYN, M.A. Fruits and vegetables juice: processing technology. West part: The AVI. Publ., 1961. 1028p. 